\title{
A Longitudinal Follow-up Study of Depression, Disability, and Body Concept in Torticollis
}

\author{
M. JAHANSHAHI and C. D. MARSDEN \\ MRC Human Movement \& Balance Unit \& Department of Clinical Neurology, Institute of \\ Neurology, The National Hospital, Queen Square, London, WC1N 3BG, UK \\ Reprint requests: $M$. Jahanshahi at above address
}

\begin{abstract}
Changes in depression, disability, body concept, and severity of head deviation were examined in a sample of 67 patients with idiopathic torticollis, who were reassessed 2 years after taking part in an initial study (before the use of botulinum toxin injections). Over the follow-up period, torticollis was unchanged in $41.8 \%$, had improved in $26.9 \%$ and deteriorated in $31.3 \%$ of cases. The overall levels of depression, disability, and body concept across the two occasions did not change. Changes in the clinical severity of torticollis over the follow-up period had a significant effect on psychological adjustment. Those whose torticollis improved were less depressed and disabled and a had a more positive body concept compared to the patients whose torticollis had worsened. Measures of illness severity had stronger associations with measures of psychological adjustment at follow-up than at the time of initial study. Longer duration of torticollis was associated with larger increases in depression and disability during the 2 years of follow-up. The results suggest that the experience of depression, disability, and negative body concept in a proportion of torticollis sufferers is a reaction to the neurological illness. A minority of the patients who remain chronically depressed are primary candidates for therapeutic intervention aiming at improving their adjustment to the illness.
\end{abstract}

\section{Introduction}

Depression secondary to neurological disorder often remains undetected, as patients do not always report their depression and neurologists do not always enquire about mood (Bridges and Goldberg, 1984). Even when detected, such secondary depression is frequently assumed to constitute an acute episode which will recover spontaneously, with the patient returning to the premorbid level of well-being. For primary depression, the available evidence shows that a substantial proportion of patients do not recover, even with appropriate treatment, but develop a chronic depressive illness (Keller et al., 1984; Scott, 1988). Furthermore, long duration of depressive illness before treatment has been found to be a major predictor of a chronic outcome (Keller et al., 1984). It is conceivable that for a proportion of the patients who experience depression following the onset of a neurological disorder, the depression will also have a chronic course, especially since the depression is likely to have remained undetected and untreated. Little information on this issue is available, as the majority of studies that have

$0953-4180 / 90 / 040233+14 \$ 3.50 / 0 \quad$ (C) 1990 CNS (Clinical Neuroscience) Publishers 
investigated depression in various neurological disorders have been crosssectional. One aim of the present longitudinal study was to examine changes in depression over time in a sample of patients with torticollis and to determine the proportion of cases with a chronic experience of depression.

In an earlier study (Jahanshahi and Marsden, 1988, 1990), patients with torticollis were found to be significantly more depressed and disabled and to have a more negative body concept, compared to an equally chronic group of cervical spondylosis sufferers. Multiple regression analysis established that disfigurement and disability were predictors of depression in torticollis. However, it was noted that cross-sectional analysis was inadequate to elucidate the precise nature of the relationship between depression, disability, disfigurement and body concept. The purpose of the present study was to examine the mutual impact of depression, disability, and body concept by comparing and contrasting their patterns of change across time. The major questions addressed were as follows:

1. What is the extent and direction of change in depression experienced by a sample of torticollis sufferers over a 2 year period?

2 How does change in depression relate to changes in the clinical status of torticollis and changes of disability and body concept across time?

\section{Method}

Subjects

The sample consisted of $35(52 \cdot 2 \%)$ males and $32(47 \cdot 8 \%)$ females. Their mean age was $53 \cdot 3$ years $(\mathrm{SD}=12 \cdot 1)$. The average duration of illness was 12.7 years (range 3 to 56.6 years). The mean age of onset of torticollis was 40.8 years $(\mathrm{SD}=14 \cdot 9)$. The mean duration of follow-up was $2 \cdot 2$ years $(\mathrm{SD}=0 \cdot 2)$ with a range of $1 \cdot 5$ to $2 \cdot 8$ years.

\section{Material}

The follow-up booklet of questionnaires included the scales used in the original study.

1. Torticollis Questionnaire This consisted of questions relating to the present status of the sufferer's torticollis and changes in it during the follow-up period. Patients rated the extent of control over their head position/ movement, and the degree of disfigurement on two separate 0 (no control, not disfigured) to 10 (complete control, extremely disfigured) rating scales. The experience and severity of cervical pain $(0=$ no pain to $5=$ severe pain $)$ at follow-up was also rated. The time period of interest was identified for the patient by providing the date of completion of the previous booklet at the top of the follow-up questionnaire.

2. Beck Depression Inventory (BDI, Beck et al., 1961) This 21 item scale measures depression. Each item consists of four statements representing 
increasing severity of depression, scored 0 to 3 . Scores range between 0 and 63 , with higher scores indicating higher levels of depression.

3. Functional Disability Questionnaire This 27-item scale was devised to assess the effects of torticollis on activities of daily living. It has been previously (Jahanshahi and Marsden, 1990) shown to have good internal consistency, construct validity, and test-retest reliability. Each item is scored on a 0 (not all affected) to 4 (severely affected) scale. The total scores (based on 24 of the 27 items selected from the reliability and validity analyses) range between 0 and 92, with higher scores indicating higher disability.

4. Body concept scale On this scale, "my body" was the concept that the patients rated on 22,7 point semantic differential scales. The internal consistency, construct and concurrent validity, and test-retest reliability of the scale have been shown to be acceptable (Jahanshahi and Marsden, 1990). Scores range between 21 and 147 (one item excluded on the basis of the validity and reliability analyses), with higher scores indicating a more negative body concept.

\section{Procedure}

Recruitment letters, explaining the aims of the follow-up study were sent out to 100 patients with spasmodic torticollis who had taken part in an initial study more than 2 years previously. Three patients were deceased (3\%), five $(5 \%)$ had changed address, four $(4 \%)$ were unwilling to participate, 10 $(10 \%)$ failed to respond to the initial recruitment letter. Questionnaires were sent to the remaining 78 patients. Completed questionnaires were received from $69(69 \%$ of the original sample). The response rate was $88.5 \%$ after taking account of patients who were deceased, had changed address, had failed to respond to the initial contact letter, or were unwilling to participate (and were not sent questionnaires). The 69 patients who participated in the follow-up study were compared with the 31 nonresponders in terms of demographic, illness-related and psychosocial measures. The responders and non-responders did not differ in terms of sex distribution, age of onset of torticollis, or duration of illness $(p>0.05)$, the responders were significantly older $(t=2 \cdot 3, \mathrm{df}=98, p=0 \cdot 03)$ than the nonresponders. There were no significant differences between the responders and non-responders in terms of degree of head control, disfigurement, depression, functional disability, or body concept at the time of initial study $(p>0.05)$. Those who participated in the follow-up study were therefore representative of the original sample. Two patients with tardive dystonia were excluded, resulting in a sample size of 67.

\section{Results}

\section{Changes in torticollis during the follow-up period}

As both the initial assessment and follow-up were carried out before 
treatment of torticollis with botulinum toxin became available, change in torticollis represents alterations in the clinical status of the disorder which were either spontaneous or brought about by treatments other than botulinum toxin. At follow-up, nine patients $(13.4 \%)$ had no or minimal head deviation. All of these nine patients also had mild torticollis at the time of initial study. A further $18(26.9 \%)$ cases considered their torticollis to be better at-follow-up compared to the initial study. The head deviation was unchanged in $19(28.4 \%)$ cases. Twenty-one $(31.3 \%)$ of the patients reported that their torticollis was worse. The overall self-reported degree of control over head position was improved at follow-up (mean $=5.5$, $\mathrm{SD}=2 \cdot 7$ ) compared to the first assessment (mean $=4 \cdot 3, \mathrm{SD}=3 \cdot 1 ; t=3 \cdot 6$, $\mathrm{df}=65, p<0.001)$. However, there was no change in perceived disfigurement across the two occasions (first assessment: mean $=4 \cdot 7, \mathrm{SD}=3 \cdot 0$; followup: mean $=4 \cdot 3, \mathrm{SD}=2 \cdot 9 ; t=0 \cdot 94, \mathrm{df}=62, p=0 \cdot 35)$. The proportion of patients who experienced cervical pain was similar on the two occasions, and the severity of cervical pain remained unaltered $(t=0.52, \mathrm{df}=45, p=0.61)$.

The effect of the change in the clinical status of torticollis at follow-up compared to the initial assessment on depression, disability, and body concept as well as on the clinical ratings are shown in Fig. $1(a-f)$. As the patients whose torticollis had improved, deteriorated, or remained unchanged (as judged by the patients' self-reports) during the follow-up interval did not differ in terms of depression, disability, body concept, disfigurement and pain severity on initial assessment, a series of one-way analyses of variance were performed on the scores at follow-up. The patients who had improved, deteriorated, or remained unchanged during the followup period, were different in terms of depression $(F(2,63)=8.4, p<0.001)$, disability $(F(2,61)=6 \cdot 6, p=0.002)$, body concept $(F(2,60)=3 \cdot 6, p=0 \cdot 03)$, disfigurement $(F(2,63)=5 \cdot 7, p=0 \cdot 006)$, and pain severity $(F(2,50)=4 \cdot 7$, $p<0.01)$ scores at follow-up. Scheffe post-hoc analyses revealed all these effects to be due to the differences between the patients who had deteriorated and those who had improved. In addition, there was a significant difference in disability at follow-up between the patients who had improved and those who had remained unchanged. The improved/deteriorated/ unchanged subgroups differed in terms of head control on initial assessment $(F(2,64)=5 \cdot 2, p=0 \cdot 008)$. Therefore, differences in head control at followup were examined through a one-way analysis of covariance with head control scores on initial assessment as the covariate. There were differences in head control at follow-up between the improved, deteriorated, and unchanged groups even after controlling for differences on initial assessment $(F(12,62)=23 \cdot 3, p<0 \cdot 001)$.

\section{Changes in depression, disability, and body concept during the follow-up period}

The mean depression, functional disability, and body concept scores of the patients on the two occasions are presented in Table 1. The overall levels of depression $(t=0.98, \mathrm{df}=64, p=0.33)$ and disability $(t=1 \cdot 4, \mathrm{df}=54$, 

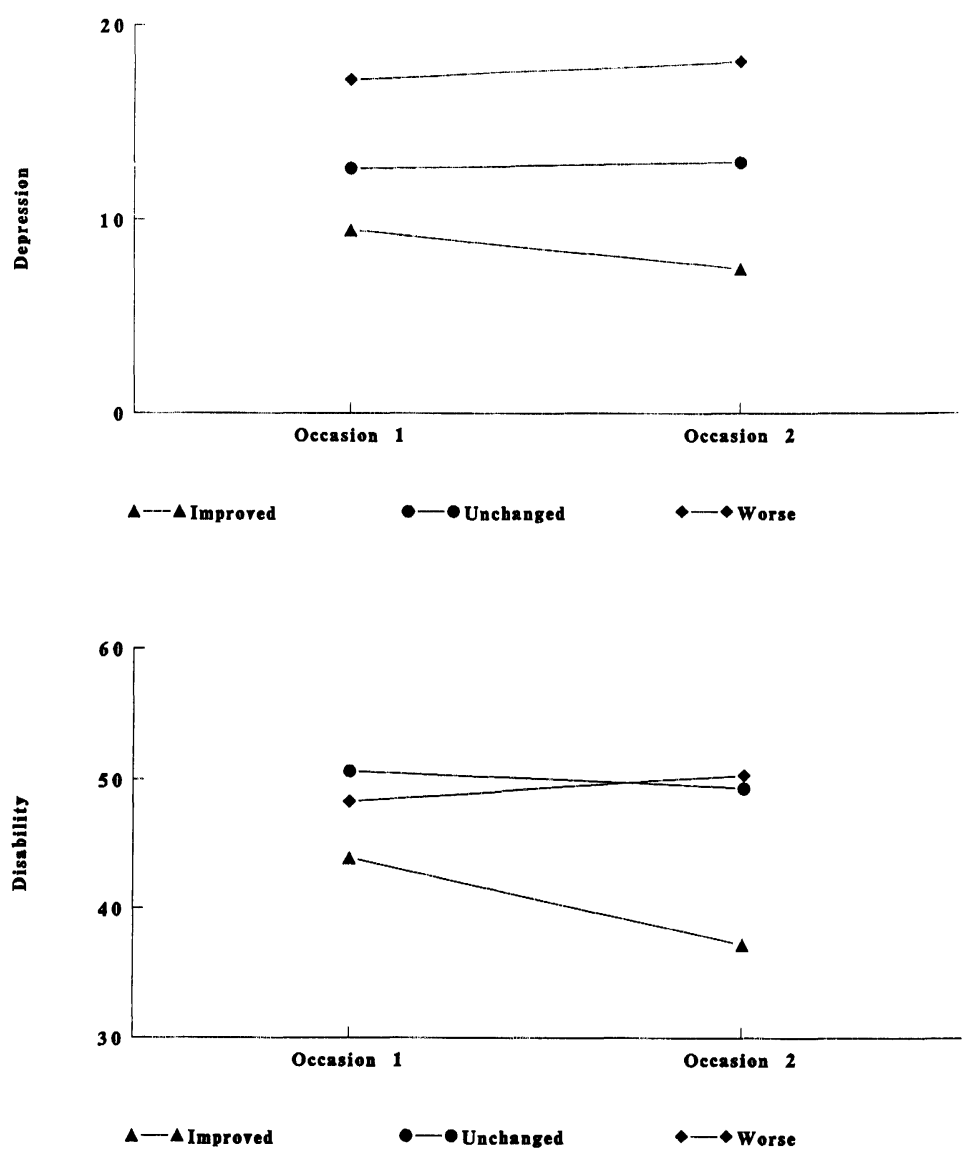

(b)

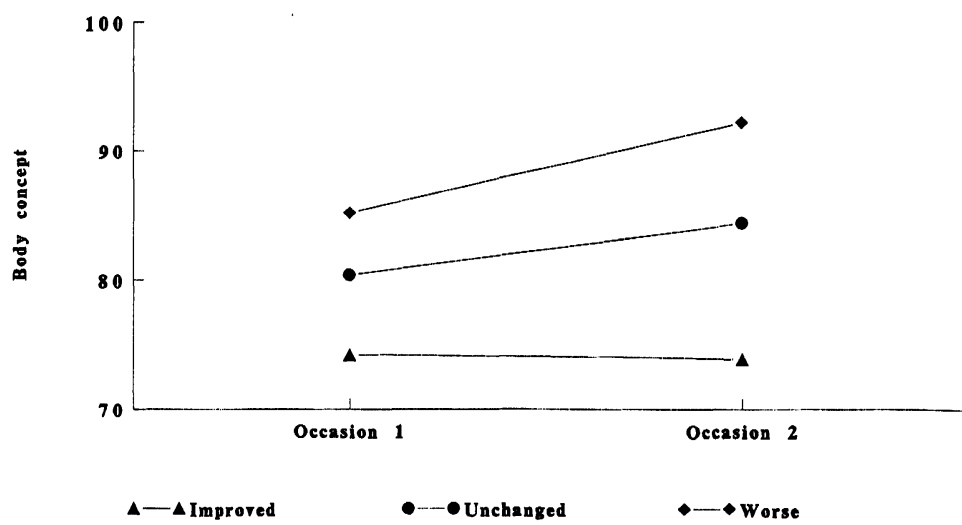

(c)

Fig. 1 a-f. The depression, disability, body concept, disfigurement, head control, and pain severity scores of the patients who reported their torticollis to be improved, deteriorated or remained unchanged at follow-up (occasion 2) compared to initial assessment (occasion 1). 

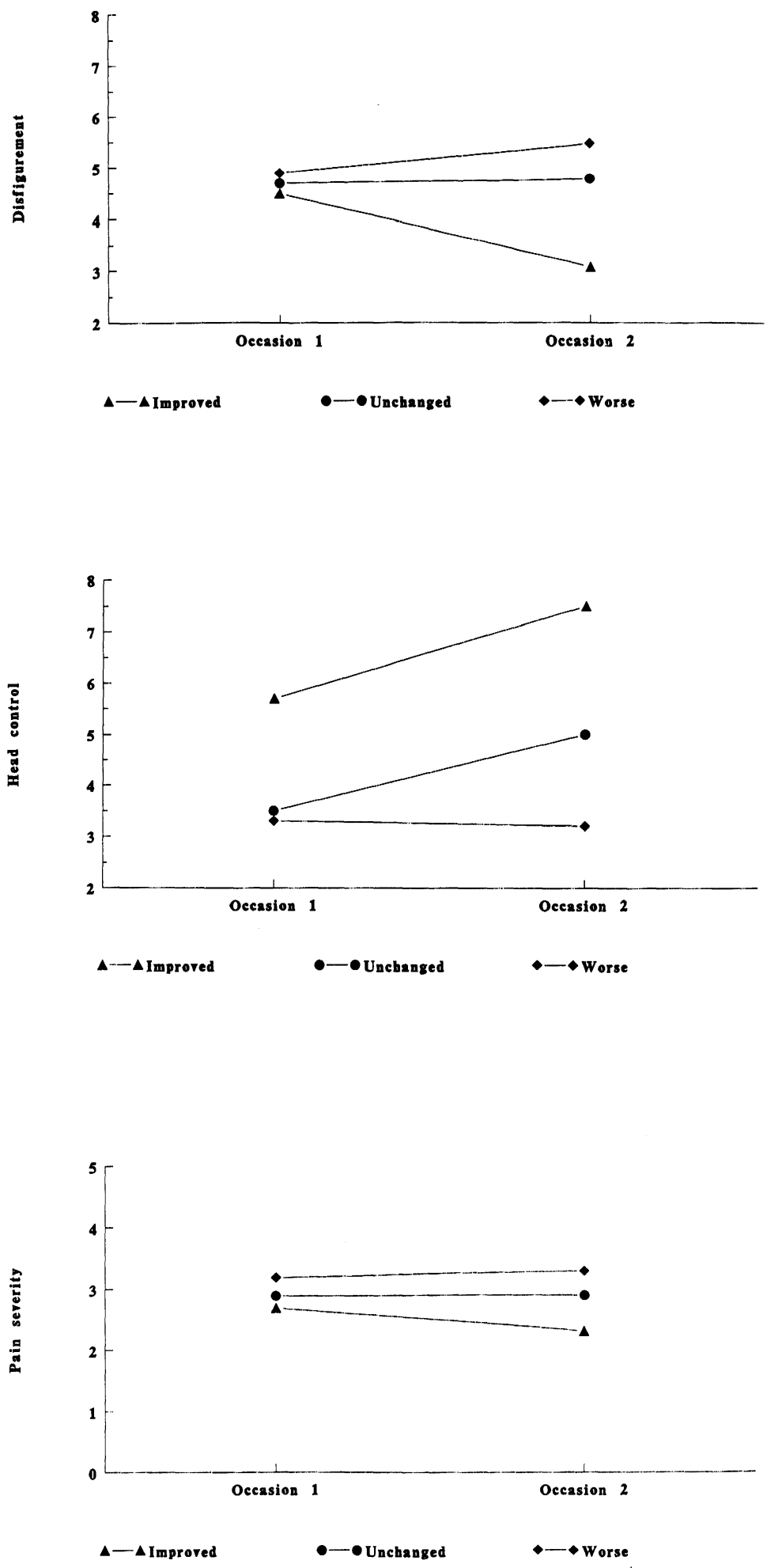
TABLE 1. Depression, disability, body concept, and disfigurement scores on the two occasions

\begin{tabular}{lccccc}
\hline & \multicolumn{2}{c}{ Initial assessment } & & \multicolumn{2}{c}{ Follow-up } \\
\cline { 2 - 3 } \cline { 5 - 6 } & Mean & $\begin{array}{c}\text { Standard } \\
\text { Deviation }\end{array}$ & & Mean & $\begin{array}{c}\text { Standard } \\
\text { Deviation }\end{array}$ \\
\hline Depression & $12 \cdot 9$ & $(10 \cdot 1)$ & & $12 \cdot 3$ & $(10 \cdot 1)$ \\
Disability & $47 \cdot 2$ & $(17 \cdot 9)$ & & $44 \cdot 4$ & $(15 \cdot 8)$ \\
Body concept & $79 \cdot 4$ & $(25 \cdot 9)$ & & $82 \cdot 6$ & $(27 \cdot 4)$ \\
\hline
\end{tabular}

$p=0 \cdot 18)$ on the two occasions were not different. Similarly, body concept $(t=1 \cdot 8, \mathrm{df}=53, p=0.09)$ was not altered.

The Pearson correlation coefficients between the first assessment and follow-up scores were 0.75 for depression, 0.61 for disability, and 0.87 for body concept. Therefore, disability showed the most and body concept the least within-subject variability across the two occasions. Nevertheless, respectively $44 \%, 63 \%$, and $34 \%$ of the variance in depression, disability, and body concept scores between the two occasions remained unaccounted for. Also, as reliance on mean scores may obscure within-subject variability across the two occasions, more detailed analysis was undertaken.

Using Beck's (1970) cut-off score of 17 for distinguishing depressed and non-depressed cases, at the first assessment $26.2 \%$ of the sample, and at follow-up $24 \cdot 2 \%$ of the patients were depressed. Forty-four patients $(67 \cdot 7 \%)$ were not depressed on both occasions. Twelve patients $(18.5 \%)$ were depressed on both occasions. Four patients $(6 \cdot 2 \%)$ who had not been depressed on first assessment were depressed at follow-up, while five cases $(7 \cdot 7 \%)$ showed the opposite pattern of change (BDI data on one or the other occasion missing for two cases).

With regard to disability, complete longitudinal information was available in 55 cases. The median score at first assessment was used to classify these patients as disabled or non-disabled on the two occasions. Forty-seven per cent of the sample were classified as disabled at first assessment, and $42 \%$ at follow-up. Across the two occasions, 20 patients $(36.4 \%)$ were nondisabled, while $15(27.3 \%)$ had remained disabled. Nine patients $(16.4 \%)$ had become more disabled during the follow-up period, whereas $11(20 \%)$ were less disabled at follow-up than on initial assessment.

Scores on the Body Concept Scale across the two occasions were relatively stable. Using a median split, $44 \%$ of the sample had a positive body concept on both occasions, compared to $48 \%$ who had a negative body concept on both occasions. In only four cases $(7 \cdot 5 \%)$, body concept had changed across the two occasions, in three cases becoming more negative at follow-up and in one patient becoming more positive. 


\section{The relationship of disability, body concept and depression across time}

For each aspect of psychological functioning, the best correlate of the score at follow-up was its score on first assessment, as shown by Pearson correlation coefficients of 0.75 for depression, 0.61 for disability, and 0.87 for body concept. Across the two occasions, the association between depression and disability had become less strong (first assessment: $r=0.63$; follow-up: $r=0.57$ ). In contrast, there was a tightening of the association of both depression (first assessment: $r=0.59$; follow-up: $r=0.72$ ) and disability (first assessment: $r=0.39$; follow-up $2 ; r=0.54$ ) with body concept, during the follow-up interval.

To assess the relationship between changes in one aspect of function with change in the other two, the inter-relations of the depression, disability, and body concept change scores were examined. The depression change score had a small and significant correlation with change in disability $(r=0.23$, $p<0.05)$, and a moderate and significant correlation with the change in body concept during the follow-up interval $(r=0.32, p=0.009)$. Change in disability and body concept were not related $(r=0 \cdot 11, p=0 \cdot 23)$.

The four subgroups differentiated on the basis of change in depression scores across the two occasions, were compared in terms of the extent and direction of change in disability and body concept. However, instead of Beck's (1974) cutoff score of 17, the median BDI score of 11 obtained at first assessment, was used to classify the sample into depressed and non-depressed groups on the two occasions. As this cut-off score was derived from the data, it allowed a more equal distribution of cases across the depressed and nondepressed groups.

The four subgroups differentiated on the basis of change in depression scores are shown in Fig. 2a. Patients in group $1(40 \%)$ were not depressed on either occasion. Group $2(12.3 \%)$ patients were depressed at initial assessment but not depressed at follow-up. The reverse pattern of change was shown by patients in group $3(13.8 \%)$, who were not depressed at initial assessment, but were at follow-up. Group $4(33.8 \%)$ consisted of patients who were depressed on both occasions.

Patients in group 1, who were not depressed on both occasions, were the least disabled on both occasions (Fig. $2 b$ ). In contrast, patients in group 4, who were depressed on both occasions, were the most disabled on both occasions. Patients in group 4, showed little alteration in the level of depression, despite a decrease $(18.4 \%)$ in disability at follow-up. Patients in groups 2 and 3 showed concordant changes in depression and disability across the two occasions. Those in group 2, who had an $8.1 \%$ decrease in disability to the level of the "non-disabled and non-depressed" group 1, also had a substantial $(55.4 \%)$ reduction of depression scores. On the other hand, the patients in group 3, showed an $8.7 \%$ increase in disability to the level of the "permanently depressed and most disabled" group 4, associated with almost a doubling (92.9\%) of depression scores from initial assessment to follow-up

Change of body concept in the four groups presented a different picture (Fig. 2c). Overall, with the exception of group 3, body concept showed little 

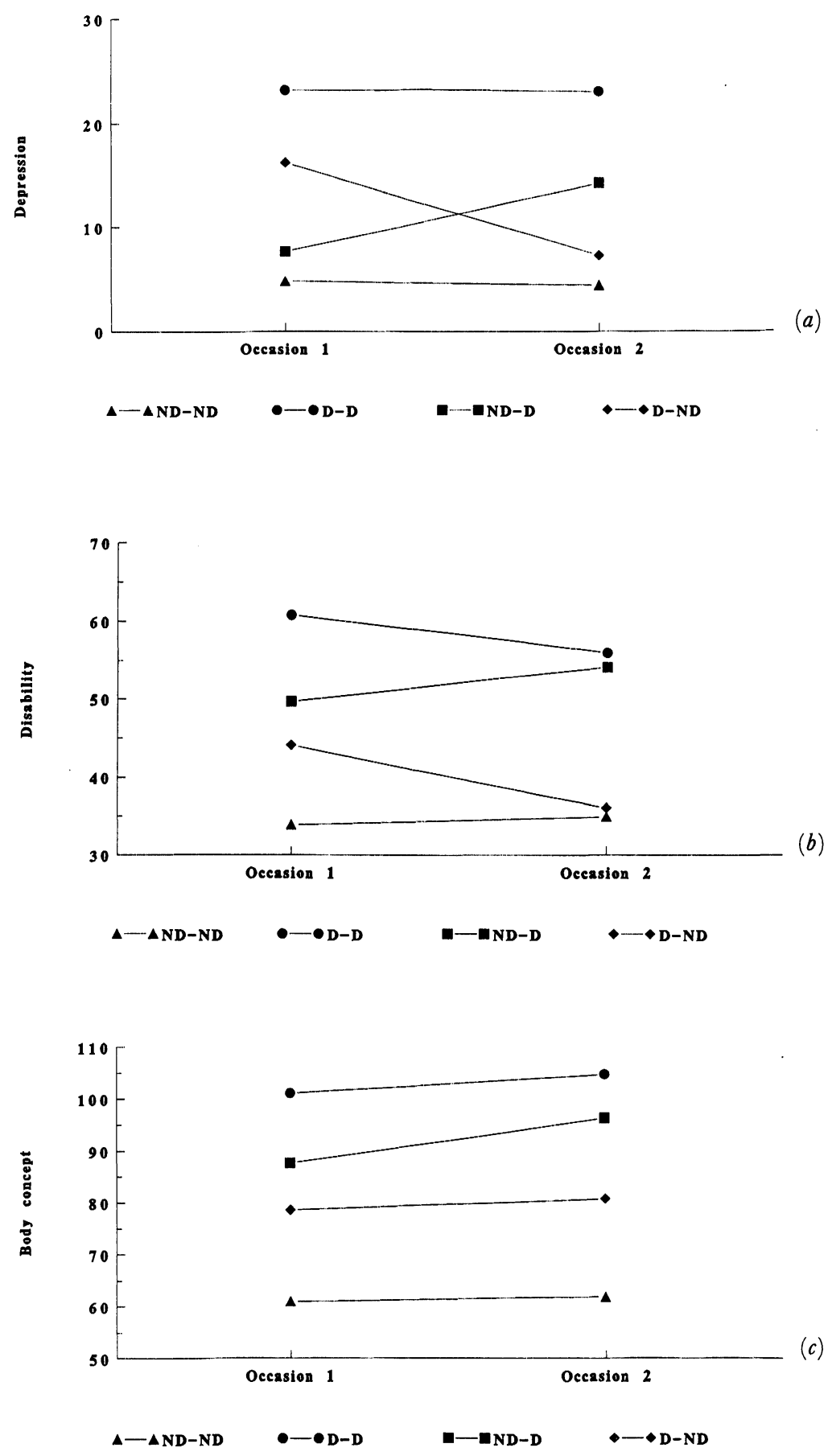

FIG. 2a-c. Initial assessment (occasion 1) and follow-up (occasion 2) scores on the depression, disability, and body concept scales for the four subgroups differentiated on the basis of change in depression scores across the two occasions defined from the median split in the Beck scores at the time of initial assessment.

ND-ND: Not depressed on both occasions (group 1)

D-D: Depressed on both occasions (group 4)

ND-D: Not depressed at initial assessment, depressed at follow-up (group 3)

D-ND: Depressed at initial assessment but not at follow-up (group 2) 
change from initial assessment to follow-up. The "permanently nondepressed" group 1, had the most positive body concept, while the "permanently depressed" group 4 had the most negative body concept across both occasions. In group 2, the reduced level of depression and disability was coupled with a slightly more negative body concept at follow-up. In contrast, the changes in the three psychological parameters were concordant in group 3: increase in disability and depression, and a more negative body concept at follow-up.

The four subgroups differentiated on the basis of the pattern of change in depression across the two occasions (Fig. 2a) did not differ in age $(F(3,63)=1 \cdot 0, \quad p=0.39)$ or sex distribution (Chi-square $=5.4, \mathrm{df}=3$, $p=0 \cdot 15)$. The four subgroups differed in terms of age of onset of torticollis $(F(3,63)=3 \cdot 3, p=0 \cdot 03)$ and duration of illness $(F(3,61)=2 \cdot 8, p=0 \cdot 04)$. The "permanently depressed" patients in group 4 and the patients in group 2 who had been depressed on first assessment but not at follow-up, had a later age of onset and shorter duration of illness than the other two groups. The clinical feature on which the four subgroups differed on both initial assessment $(F(3,58)=6 \cdot 2, \quad p<0 \cdot 001)$ and at follow-up $(F(3,60)=5 \cdot 0$, $p=0.004)$ was the extent of disfigurement. The four groups also differed in terms of head control at follow-up $(F(3,60)=3.9, p<0.01)$ and pain severity on occasion $1(F(3,46)=4 \cdot 7, p<0 \cdot 01)$. Post-hoc Scheffe tests revealed that the differences in the clinical features of torticollis resulted from the "permanently depressed" group 4 being significantly more disfigured, having less head control and more severe pain than the "permanently non-depressed" group 1.

\section{The inter-relations of depression, disability, and body concept with demographic and clinical features}

Table 2 shows the Pearson correlation coefficients of age, age of onset, duration of illness and illness-related factors (degree of head control, perceived disfigurement, and cervical pain severity) with depression, disability, and body concept on initial assessment and at follow-up. Besides the significant associations of depression with age and age of onset on initial assessment, which were no longer present at follow-up, depression, disability, and body concept scores were not significantly related to age, age of onset, or duration of illness on either occasion. Overall, the association between the clinical features of torticollis (degree of head control, disfigurement, and cervical pain severity), and depression, disability, and body concept was stronger at the follow-up than at first assessment.

The association of change in the clinical features of torticollis with change in depression, disability, and body concept was also examined. Change in disability was correlated with change in head control $(r=0.46$, $p<0.001)$, disfigurement $(r=0.36, p=0.003)$, and pain severity $(r=0.47$, $p=0.002)$. Change in depression showed small correlations with disfigurement $(r=0 \cdot 18, p=0.09)$, pain severity $(r=0.24, p=0.06)$, and head control $(r=0 \cdot 16, p=0 \cdot 10)$, all of which failed to attain significance at the $5 \%$ level. 
TABLE 2. Pearson correlation coefficients between the demographic and clinical variables and depression, disability, and body concept on initial assessment and at follow-up.

\begin{tabular}{|c|c|c|c|c|c|c|}
\hline & \multicolumn{3}{|c|}{ Initial assessment } & \multicolumn{3}{|c|}{ Follow-up } \\
\hline & Depression & Disability & $\begin{array}{c}\text { Body } \\
\text { Concept }\end{array}$ & Depression & Disability & $\begin{array}{c}\text { Body } \\
\text { Concept }\end{array}$ \\
\hline Age & $\cdot 22 \dagger$ & -.01 & -.06 & $\cdot 12$ & -.02 & -.03 \\
\hline Age of onset & $.29 \dagger$ & .02 & -.01 & $\cdot 11$ & $-\cdot 13$ & .06 \\
\hline $\begin{array}{l}\text { Duration of } \\
\text { illness }\end{array}$ & -.14 & -.07 & $\cdot 01$ & .01 & $\cdot 16$ & -.04 \\
\hline Disfigurement & $.44 *$ & $.52 *$ & $.49 *$ & $.50 *$ & $.68 *$ & $.61 *$ \\
\hline $\begin{array}{l}\text { Degree head } \\
\text { control }\end{array}$ & $-\cdot 36^{*}$ & $-\cdot 47 *$ & $-\cdot 05$ & $-\cdot 49 *$ & $-\cdot 40^{*}$ & $-.41 *$ \\
\hline Pain severity & $.47 *$ & $\cdot 33 \dagger$ & $\cdot 25$ & $\cdot 41^{*}$ & $\cdot 49^{*}$ & $\cdot 29 \dagger$ \\
\hline
\end{tabular}

Change in body concept across the two occasions showed a small and near significant association with change in disfigurement $(r=0 \cdot 19, p=0.09)$, but was not related to change in head control $(r=-0 \cdot 14, p=0 \cdot 16)$, or pain severity $(r=0.09, p=0.29)$. Duration of illness and age of onset were associated with the change scores of depression (respectively, $r=0.25$, $p=0.02 ; r=-0.25, p=0.02$ ), disability (respectively, $r=0.28, p=0.02$; $r=-0.23, p=0 \cdot 04$ ), and pain severity (respectively, $r=0.28, p=0.03$; $r=-0 \cdot 26, p=0 \cdot 04)$.

\section{Discussion}

\section{Change in torticollis}

In the course of the two years of the follow-up, $26.9 \%$ of the patients considered their torticollis to have improved, $31.3 \%$ were worse, while $41.8 \%$ reported their head deviation to be unchanged (including the nine with minimal head deviation on both occasions). Although the change in the clinical status of torticollis over the follow-up period was based on the patients' own evaluations, evidence for its concurrent validity is available. The patients whose torticollis had deteriorated over the follow-up period had less head control, more disfigurement and higher pain severity at followup compared to the patients who had improved, despite their initial similarity in these clinical features on first assessment.

The patients whose torticollis had improved or deteriorated over the follow-up period, did not differ in terms of depression, disability, and body concept on initial assessment, but the "deteriorated" group were more disabled and depressed and had more negative body concept at follow-up than the "improved" group (Fig. 1 $a-c$ ). The fact that the change in the clinical status of torticollis across the two occasions had a significant effect on 
the psychological parameters at follow-up, suggests that depression, disability, and negative body concept may be reactive in nature. This interpretation is also supported by the fact that change in disability across the two occasions was associated with change in disfigurement, head control, and pain severity. The associations between the depression change score and the change in the clinical features of torticollis, however, only approached significance, suggesting that the impact of the changes in the clinical features of torticollis on the experience of depression may be indirect, via the resulting alterations of disability.

\section{Change in depression, disability and body concept}

The absence of change in the clinical status of torticollis for the greatest proportion $(42 \%)$ of patients was also reflected in the lack of significant overall change in depression, disability or body concept. Nevertheless, more detailed examination of the data revealed some within-subject variability in psychological functioning across the two occasions. Disability showed the greatest, body concept the least, and depression intermediate degrees of within-subject variability across the two occasions. Thirty-six per cent of the sample had considerable change in their disability status across the two occasions, either becoming more or less disabled. In contrast, only $7.5 \%$ of the patients showed change of body concept from positive to negative or the reverse pattern across occasions. Using Beck's (1970) cut-off score, 14\%, and using a median split, $26 \%$ of the patients had shifted from a depressed to a non-depressed status, or from a non-depressed to a depressed status during the follow-up period.

The pattern of correlations suggests that with greater chronicity of illness, more severe torticollis is increasingly associated with poorer psychological adjustment in a more direct fashion. Despite the lack of a significant linear relationship between duration of illness and the measures of psychological adjustment on either first assessment or at follow-up, duration of illness had positive and significant associations with change in depression $(r=0.25, p=0.02)$ and disability $(r=0.28, p=0.02)$ across the two occasions, such that greater chronicity was associated with greater increases in depression and disability during the 2 years of follow-up.

Although the small and significant associations between the depression change score and change in disability and body concept across the two occasions can be interpreted as suggestive of a simple causal link between depression and these two aspects of psychological functioning, other findings indicate that the relationship between depression and disability, or depression and body concept is not unidirectional or static across time or uniformly concordant for all sufferers.

The conclusion that the relationship between the three psychological parameters was not static across time was demonstrated by the fact that the major "determinants" of depression and disability across the two occasions were different. At initial assessment, depression and disability were the highest correlates of each other. At follow-up, depression had become most 
highly correlated with body concept, while disability had its highest correlation with disfigurement. In contrast, on both occasions, disfigurement and depression were the major correlates of body concept.

Furthermore, the four subgroups defined on the basis of change in depression, did not always show concordant changes in disability or body concept across time (Fig. $2 a-c$ ) as would be expected from a simple linear causal model. For example, the $14 \%$ of cases in group 3 who had become more disabled and depressed during the follow-up period, had a less negative body concept at follow-up. Similarly, the $33.8 \%$ of cases in group 4 who were depressed on both occasions, showed little alteration in depression or body concept during the follow-up despite an $18.4 \%$ decrease in disability.

This latter "permanently depressed" group meets the criterion for chronic depression defined as "persistent symptoms for two or more years" (Cassano et al., 1983; Scott, 1988). Using Beck's cut-off score of 17, 18.5\%, and using a median split, $33.8 \%$ of the sample suffered from chronic depression. This group consisted of those patients who had failed to adjust to the illness: they were the most disabled and had the most negative body concept. These patients were therefore victims of psychological morbidity, which the longitudinal follow-up showed to persist over time. At the other extreme, the "permanently not depressed" group 1 consisted of patients with low levels of depression and disability and a positive body concept on both occasions representing those who had adjusted well to the illness. The differences between these two groups which represent the two extreme patterns of adjustment to illness, may provide clues as to the factors that render individuals vulnerable to psychological morbidity in the course of torticollis. There were no age or sex differences between the two groups. The "permanently depressed" group had a later age of onset and shorter duration of illness than the "permanently non-depressed" group although not significantly so. Amongst the variables measured, the various indices of the severity of torticollis were the factors that differentiated the two groups. The "permanently depressed" were more disfigured, had less head control and more severe cervical pain than the "permanently non-depressed". It therefore seems that the greater depression, disability, and negative body concept of the "permanently depressed" group results from the greater severity of their illness.

Therefore, various aspects of the longitudinal and correlational data indicate that the experience of depression by torticollis sufferers may be reactive and secondary to the neurological disorder. This has implications for the treatment of depression. Although the course and outcome of secondary depression is considered to largely depend on changes in the primary causal factor (Jablensky, 1987), decisions regarding the management of secondary depression should depend on whether the depression is secondary to an acute or chronic medical disorder. In the depressed acutely ill medical patient it can be assumed that the depression will lift with recovery from the medical illness. Within the context of chronic neurological disorders such as torticollis, where there is less chance of spontaneous 
remission or treatment-related recovery, this assumption is not warranted, and direct management of depression is necessary. In the present sample this point was well demonstrated by the "permanently depressed group" who had remained depressed during the 2 years of the follow-up period. These patients with chronic depression are candidates for treatment. Besides attempts at better management of their torticollis which would be expected to have an ameliorating effect on their depression, cognitive-behavioural treatment to improve these patients' adjustment to their illness would be appropriate.

\section{Acknowledgements}

This work was supported by grants from the Medical Research Council and the Dystonia Society.

\section{References}

Beck, A. T. (1970). "Depression". University of Pennsylvania Press, Philadelphia.

Beck, A. T., Ward, C. H., Mendelson, M., Mock, J. E. and Erbaugh, J. K. (1961). An inventory for measuring depression. Archives of General Psychiatry, 4, 561-571.

Bridges, K. W. and Goldberg, D. P. (1984). Psychiatric illness in inpatients with neurological disorders: patients' views on discussion of emotional problems with neurologists. British Medical Journal, 289, 656-658.

Cassano, G. B., Maggini, C. and Akiskal, H. (1983). Short-term, subchronic and chronic sequelae of affective disorders. The Psychiatric Clinics of North America, 6, 55-68.

Jablensky, A. (1987). Editorial: Prediction of the course and outcome of depression. Psychological Medicine, 17, 1-9.

Jahanshahi, M. and Marsden, C. D. (1988). Depression in torticollis: A controlled study. Psychological Medicine, 18, 925-933.

Jahanshahi, M. and Marsden, C. D. (1990). Body concept, depression and disability in patients with spasmodic torticollis. Behavioural Neurology, 3, 117-131.

Keller, M. B., Klerman, G. L., Lavori, P. W., Coryell, W., Endicott, J. and Taylor, J. (1984). Long-term outcome of episodes of major depression. Journal of the American Medical Association, 252, 788-792.

Scott, J. (1988). Chronic depression. British Journal of Psychiatry, 153, 287-297. 


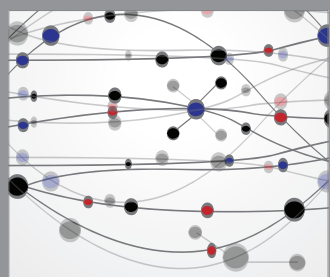

The Scientific World Journal
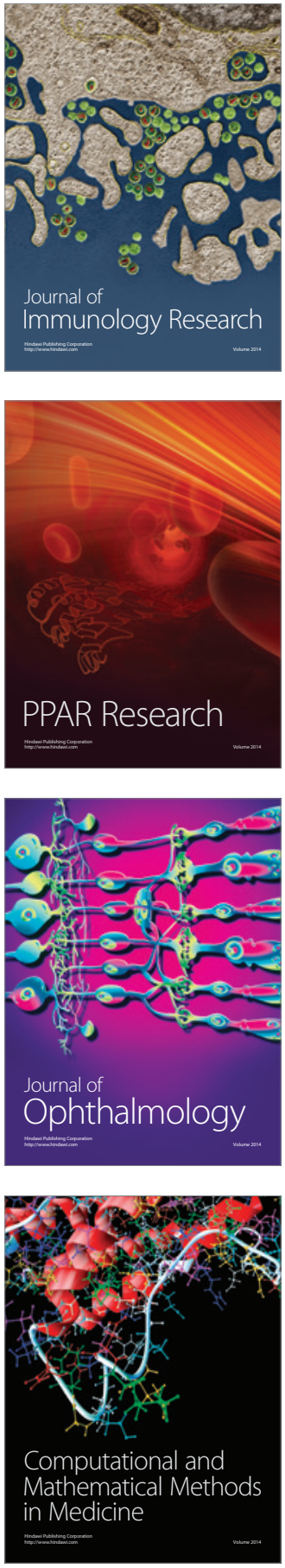

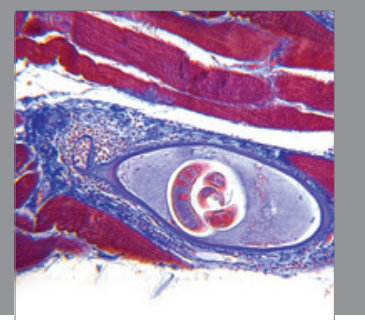

Gastroenterology

Research and Practice
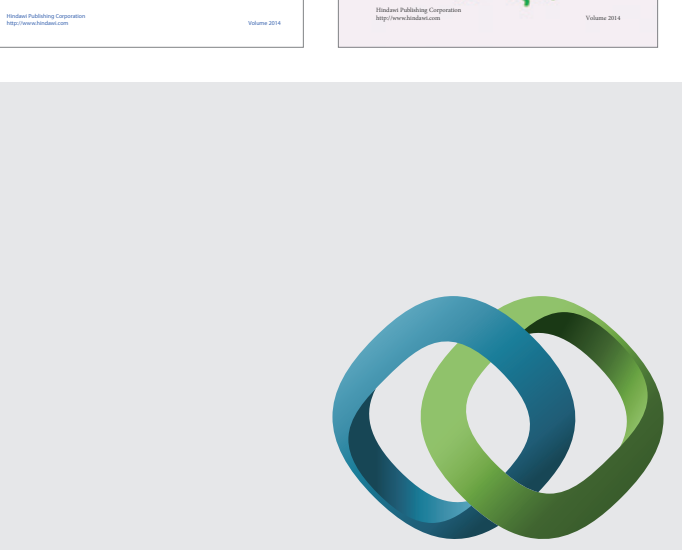

\section{Hindawi}

Submit your manuscripts at

http://www.hindawi.com
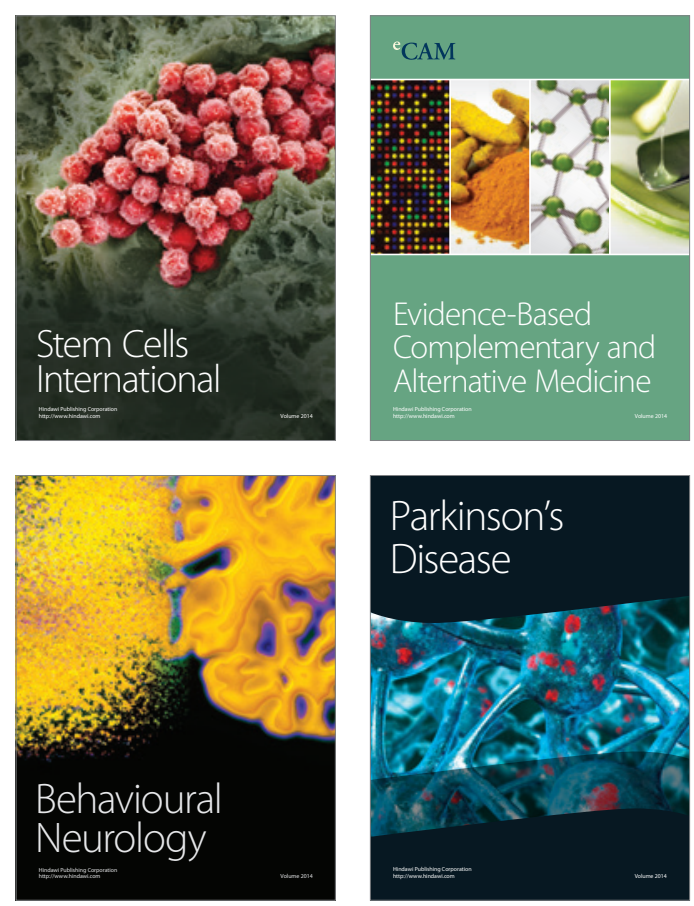

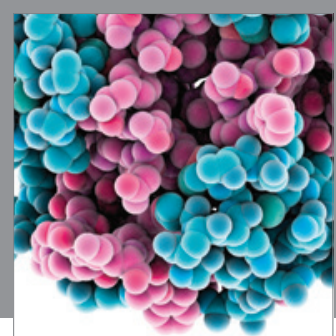

Journal of
Diabetes Research

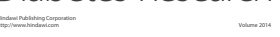

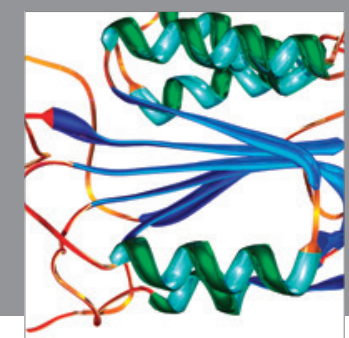

Disease Markers
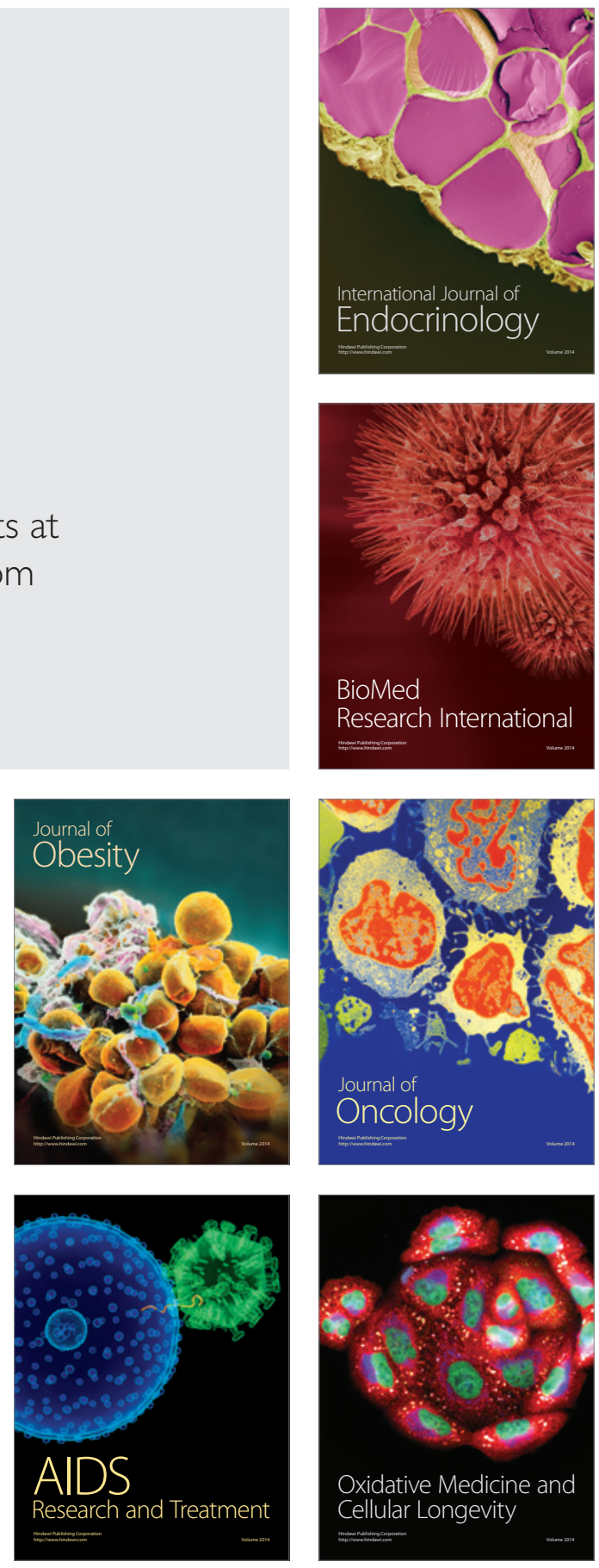